\title{
Steuerung der Migrationsströme : Überlegungen zur französischen Politik des "codéveloppement“
}

Jérôme Audran

\section{OpenEdition}

1 Journals

Electronic version

URL: http://journals.openedition.org/sjep/323

DOI: $10.4000 /$ sjep.323

ISSN: 1663-9677

Publisher

Institut de hautes études internationales et du développement

Printed version

Date of publication: 1 décembre 2008

Number of pages: 107-121

ISBN: 978-2-940415-08-3

ISSN: 1660-5926

\section{Electronic reference}

Jérôme Audran, «Steuerung der Migrationsströme : Überlegungen zur französischen Politik des „Codéveloppement" », Schweizerisches Jahrbuch für Entwicklungspolitik [Online], 27-2 | 2008, Online erschienen am: 31 Mai 2010, abgerufen am 08 September 2020. URL : http://journals.openedition.org/ sjep/323 ; DOI : https://doi.org/10.4000/sjep.323 


\title{
Steuerung der Migrationsströme: Überlegungen zur französischen Politik des "codéveloppement"
}

\author{
Jérôme Audran*
}

W ie in den meisten entwickelten Ländern hat auch in Frankreich die Migrationspolitik in den vergangenen Jahrzehnten einen Wandel durchlaufen. Traditionellerweise in sicherheitspolitischen Anliegen verwurzelt, hat sie sich nach und nach für Entwicklungsfragen geöffnet. Entsprechend dieser neuen Ausrichtung bedient sie sich der öffentlichen Entwicklungshilfe (aide publique au développement, APD) als Mittel, um den vom Süden ausgehenden Migrationsdruck auf den Norden abzuschwächen. Indem sie die APD in den Dienst einer wirksameren Steuerung der Migrationsströme stellt, schlägt die französische Einwanderungspolitik eine neue Richtung ein: Nach dem kläglichen Scheitern der Bestrebungen, die illegale Einwanderung durch Repression einzudämmen, setzt sich heute nach und nach die Erkenntnis durch, dass der Kampf gegen die illegale Migration nur über die Entwicklung der Herkunftsländer gewonnen werden kann ${ }^{1}$. Die ursprünglich rein repressive Strategie wird damit um eine präventive Stossrichtung erweitert.

Offenkundig wurde diese Instrumentalisierung der APD im Mai 2007 mit der Schaffung des französischen Ministeriums für Immigration, Integration, nationale Identität und solidarische Entwicklung (Ministère de l'Immigration, de l'Intégration, de l'Identité nationale et du Codéveloppement). Die Erwähnung des codéveloppement in der Bezeichnung dieses neuen Ministeriums ist kein Zufall, sondern Ausdruck der Entschlossenheit des neu gewählten französischen Staatspräsidenten, der Entwicklungshilfe eine neue Ausrichtung und eine neue Dimension zu verleihen². Durch seine Beteiligung bei der „Definition und Umsetzung von Kooperations- und Entwicklungshilfepolitiken, die zur Migrationssteuerung beitragen“"3, bestärkt dieses neue staatliche Organ die Überzeugung, Entwicklung und Migration stünden in einem engen Zusammenhang zueinander, und symbolisiert die ideologische Verknüpfung von Entwicklungsfragen mit der wachsenden Herausforderung der Migrationsproblematik.

Diese Verknüpfung hat ihren Ursprung in einer in politischen Kreisen weit verbreiteten Auffassung, wonach die internationale Migration letztlich durch Armut

* Diplom des Institut de hautes études internationales et du développement IHEID (Master in International Affairs) und Mikrofinanz-Analyst bei Symbiotics (Genf).

1 H. de Haas, Turning the Tide? Why „Development Instead of Migration“ Policies are Bound to Fail, Oxford, International Migration Institute, University of Oxford, 2006.

2 B. Hortefeux, „Le codéveloppement, un enjeu majeur pour la France“, Les Echos, 31. August 2007.

3 Décret no 2007-999 du 31 mai 2007 relatif aux attributions du ministre de l'Immigration, de l'Intégration, de l'Identité nationale et du Codéveloppement, Journal officiel de la République française, Nr. 125, 1. Juni, S. 9964, Text Nr. 11 (eigene Übersetzung). 
hervorgerufen wird ${ }^{4}$. Für den Vorsteher des neuen Ministeriums, Brice Hortefeux, besteht über den Auslöser der Migration kein Zweifel ${ }^{5}$ : Der Einwanderungsdruck auf die Länder des Nordens sei in erster Linie auf die Ungleichgewichte im Süden zurückzuführen. Gegenwärtig stammen allein 65 Prozent der Einwanderer, die auf reguläre Weise nach Frankreich gelangen, aus Afrika. Die illegale Einwanderung könne daher nur eingedämmt werden, indem das Problem an seiner Wurzel, sprich an der Unterentwicklung, gepackt werde. In anderen Worten: Frankreich müsse „die Entwicklung der Herkunftsländer unterstützen, indem die Frage der Migration verstärkt als bisher berücksichtigt wird“6. Hortefeux will sich aktiv an der Ausarbeitung und Umsetzung einer Entwicklungshilfepolitik beteiligen, die auf die Herkunftsländer der Migrantinnen und Migranten ausgerichtet ist. Dabei hat er ein klares Ziel vor Augen: Frankreich soll in Europa eine Vorreiterrolle einnehmen in Bezug auf die Konzipierung und Umsetzung wirksamer Instrumente der öffentlichen Entwicklungshilfe, welche die Migrantinnen und Migranten in die Entwicklung ihrer Herkunftsländer einbinden, damit die Menschen in den Auswanderungsländern bessere Lebensbedingungen vorfinden und nicht mehr anderswo ums Überleben kämpfen müssen?

Damit ist die französische Einwanderungspolitik untrennbar mit Entwicklungsfragen verknüpft. Dieses Phänomen lässt sich übrigens auch in anderen Industrieländern oder auf der Ebene der Europäischen Union beobachten, welche namentlich über ihr Programm MEDA ${ }^{8}$ die Spirale von geringem Wachstum, Arbeitslosigkeit, Armut und Migration durchbrechen will. Diese Annäherung von Entwicklungszusammenarbeit und Migrationspolitik wirft natürlich verschiedene Fragen in Bezug auf die Zweckmässigkeit eines solchen Vorgehens auf. Ist die von der französischen Regierungspolitik beschworene Beziehung zwischen der Entwicklung der Herkunftsländer und der Migrationssteuerung tatsächlich vorhanden? Kann der Migrationsdruck durch Entwicklung beziehungsweise durch Armutsverringerung effektiv verringert werden? Wäre es nicht sinnvoller, die Fragenkomplexe der internationalen Migrationsbewegungen auf der einen und der Entwicklung der Herkunftsländer auf der anderen Seite unabhängig voneinander zu betrachten? Um diese Fragen zu beantworten, müssen verschiedene Aspekte vertieft untersucht werden. Im Folgenden soll zunächst die Entstehung des Konzepts des codéveloppement historisch nachvollzogen werden, denn nur so lassen sich die ihm beigemessene Bedeutung, seine Ziele und Herausforderungen und seine schrittweise Institutionalisierung auf den höchsten staatlichen Ebenen Frankreichs adäquat erfassen. Anschliessend werden die verschiedenen Instrumente analysiert, die bei der Umsetzung der Politik des codéveloppement zum Einsatz kommen, bevor abschliessend die Zweckmässigkeit, die Vor- und Nachteile sowie die Risiken der Instrumentalisierung der APD im Kampf gegen die illegale Einwanderung erörtert werden.

4 P. Stalker, „Migration Trends and Migration Policy in Europe“, International Migration, vol. 40, Nr. 5, S. 151-179.

5 B. Hortefeux, op. cit.

6 Ansprache von Brice Hortefeux anlässlich der „Journées de la coopération internationale et du développement“, 18. Juli 2007, <http://www.immigration.gouv.fr/article.php?id_article=85>.

B. Hortefeux, „Le codéveloppement, un enjeu majeur pour la France“, op. cit.

8 Ziel des Programms MEDA ist es, Massnahmen der Zusammenarbeit durchzuführen, die Drittstaaten im Mittelmeerraum bei der Reform ihrer wirtschaftlichen und sozialen Strukturen unterstützen und die Auswirkungen der wirtschaftlichen Entwicklung auf Gesellschaft und Umwelt abfedern (<http://europa.eu $>$ ). 
Das Konzept des codéveloppement ist relativ neu. Es wurde von der französischen Entwicklungshilfe geprägt und bereits mehrfach in der Praxis angewendet. Sein Vormarsch begann mit der Machtergreifung der Linken in den 1980erJahre. Das damalige Ziel war äusserst ehrgeizig: Zwischen Frankreich und gewissen privilegierten Partnern aus den Rängen der Entwicklungsländer sollte eine echte solidarische Entwicklung - ein co-développement eben - stattfinden, die auf der Komplementarität Frankreichs und dieser Länder aufbaut (Knowhow und Spitzentechnologie auf der einen, Arbeitskräfte und natürliche Rohstoffe auf der anderen Seite). Anders ausgedrückt: Die französische Entwicklungshilfepolitik strebte danach, mit den Ländern des Südens eine gerechte Verteilung des weltweiten Reichtums auszuhandeln. Wie Christophe Daum ${ }^{9}$ ausführt, wurde diese hehre Utopie von der Realpolitik jedoch sehr rasch zerschmettert, und die Kluft zwischen Nord und Süd vertiefte sich weiter, anstatt sich zu verringern.

Eine Verknüpfung des codéveloppement mit Migrationsfragen stand damals nicht zur Debatte, denn die APD betraf nur Länder, die eigens zu diesem Zweck bilaterale Verträge mit Frankreich geschlossen hatten. Vor allem aber war die Migrationssteuerung für die Industrieländer - darunter auch Frankreich damals kein Thema, denn die Süd-Nord-Migration wurde nicht als Bedrohung wahrgenommen. Seither ist die Einwanderung zu einer zentralen innenpolitischen Frage geworden. Im Brennpunkt steht nicht mehr nur die Integration von Migrantinnen und Migranten in der Gastgesellschaft, sondern auch die Aufnahme von Neuankömmlingen, was durch die Bewegung der Sans-papiers 1997 offensichtlich wurde. In diesem Kontext bot sich das codéveloppement als zweckmässige Antwort an, die den Problemen der Einwanderung und der Entwicklung gleichermassen gerecht wird. Anfang der 1990er-Jahre wurden im Tal des Senegal-Flusses (im Westen Malis, im Norden Senegals und im Süden Mauretaniens) erste konkrete Versuche unternommen. Diese Erfahrungen haben die Definition des Konzepts in seiner heute akzeptierten Form massgeblich geprägt. Konkret ging es darum, über eine Nichtregierungsorganisation rückkehrwillige Migrantinnen und Migranten mit Finanzhilfen und Begleitmassnahmen beim Aufbau einer Wirtschaftstätigkeit zu unterstützen.

Die noch heute verbindliche Definition des codéveloppement wurde 1998 vom damaligen Innenminister Jean-Pierre Chevènement explizit formuliert. Sie war nicht minder ehrgeizig als jene, die zu Beginn der 1980er-Jahre vorherrschte, wich aber in verschiedenen Punkten davon ab. Das Ziel lautete, das Fachwissen und die finanziellen Kapazitäten der Migrantinnen und Migranten in den Dienst der Entwicklung ihrer Herkunftsländer zu stellen. Die Parallelen zum heutigen Verständnis des codéveloppement sind unverkennbar. In der Praxis allerdings orientierten sich die Massnahmen nach wie vor an den Erfahrungen, die in den vergangenen Jahren im Bereich der Rückkehrhilfe gemacht wurden, und beschränkten sich letztlich darauf, diese zu optimieren. Diese Fokussierung auf die Rückkehr ging einher mit der impliziten Absicht, die Gewährung von Krediten für Massnahmen des codéveloppement an greifbare Erfolge in Bezug auf die

9 C. Daum, „Le codéveloppement, grandeur et décadence d'une aspiration généreuse“, Revue internationale et stratégique, Nr. 4 (68), 2007, S. 49-59. 
Eindämmung der illegalen Einwanderung zu knüpfen. Die Partnerländer, die an der Durchführung von Pilotversuchen beteiligt werden sollten (Mali, Senegal, Marokko und Komoren), reagierten auf die für die Rückkehr gesetzten Ziele (25000) skeptisch und lehnten das Vorhaben teilweise gar $\mathrm{ab}^{10}$.

Im heutigen politischen Diskurs bezeichnet codéveloppement die Gesamtheit der Entwicklungshilfemassnahmen, die in einem Zusammenhang mit den Einwanderern und der Entwicklung ihrer Herkunftsländer stehen ${ }^{11}$. Diese relativ weit gefasste Definition wurde von den aufeinander folgenden Regierungen mit geringfügigen Änderungen übernommen. Im Dezember 2006 präzisierte die ehemalige Ministerin für Entwicklungsfragen, Brigitte Girardin, das Konzept wie folgt: „Das codéveloppement lässt sich relativ einfach definieren. Es umfasst sämtliche Entwicklungsmassnahmen, an der in Frankreich lebende Migrantinnen und Migranten beteiligt sind." ${ }^{2}$ Von den Massnahmen des codéveloppement können sowohl Einwanderer profitieren, die in ihr Herkunftsland zurïckkehren möchten, um sich dort eine Existenz aufzubauen, aber auch Geschäftsleute, Wissenschaftler, Ärzte und Ingenieure, die zwar ihren Wohnsitz in Frankreich beibehalten wollen, aber bereit sind, in Produktionsanlagen oder soziale Projekte in ihrem Herkunftsland zu investieren (Schulen, Gesundheitseinrichtungen usw.) oder ihre Kompetenzen, ihr Fachwissen und ihr Beziehungsnetz dem Land zur Verfügung zu stellen ${ }^{13}$.

Obwohl in der Zwischenzeit formell von dem Ziel der Migrationssteuerung abgerückt wurde, wird auch heute noch erwartet, dass das codéveloppement zur Eindämmung der Migration beitragen kann. Dies lässt sich zumindest in der Praxis feststellen. Der Grundgedanke lautet, dass die von den Auswanderern bewirkten Entwicklungsfortschritte in den Ländern des Südens den Migrationsdruck verringern, weil sich Migrationswillige aufgrund der sich verbessernden Lebensbedingungen eher für einen Verbleib in ihrem Land als für eine Auswanderung entscheiden. In anderen Worten: Die heutigen Einwanderer in Frankreich tragen zur Verbesserung des Lebensstandards in ihren Herkunftsländern bei und sorgen so dafür, dass in Zukunft weniger Menschen auswandern. Ausserdem bedient sich das codéveloppement der APD mit dem Ziel, die Migrationsströme zu steuern. Tatsächlich knüpft Frankreich einen Teil seiner APD an gewisse Bedingungen in Bezug auf die Wirksamkeit der Migrationspolitik der Entwicklungsländer. Ganz abgesehen von der erklärten Absicht der Regierung, die Herkunftsländer bei ihrer Entwicklung zu unterstützen, verkörpert der Begriff codéveloppement auch heute noch einen ausgeprägten politischen Willen, die Migrationströme zu kontrollieren.

Aus institutioneller Sicht wurde mit der Errichtung des neuen Ministeriums für Einwanderung auf höchster staatlicher Ebene ein Forum für Entwicklungs- und

10 J. Godfrain et R. Cazenave, Rapport sur le codéveloppement, rapport envoyé au Ministère des affaires étrangères de la France, Januar 2007, $<\mathrm{http}: / /$ www.richardcazenave.com/media/Rapport_codeveloppement.pdf $>$.

11 S. Naïr, Rapport de bilan et d'orientation sur la politique de codéveloppement liée aux flux migratoires, Paris, Mission interministérielle „migrations/codéveloppement“, Ministère des affaires étrangères, 1997.

12 Discours de la ministre déléguée à la Coopération, au Développement et à la Francophonie, $M^{m e}$ Brigitte Girardin, Forum national des acteurs du codéveloppement, Paris, 18. Dezember 2006.

13 Ministère des affaires étrangères (MEA), Le codéveloppement: présentation générale, Paris, MEA, 26. Januar 2005. 
Migrationsfragen geschaffen. Erstmals wurde damit die Zuständigkeit für die Einwanderungspolitik einem einzigen vollwertigen Ministerium übertragen, welches ausserdem für das Asylwesen und für Einbürgerungen verantwortlich ist $^{14}$. Die Aufmerksamkeit, die der Einwanderungsproblematik gewidmet wird, zeugt von der Bedeutung dieses Themas für die Wählerschaft. Tatsächlich bildete die Einwanderungsfrage im Jahr 2007 einen Schwerpunkt des Wahlkampfs von Nicolas Sarkozy um das Präsidentenamt. Das neue Ministerium ist insbesondere an der Ausarbeitung und Umsetzung der Entwicklungshilfepolitiken beteiligt, die einen Beitrag zur Migrationsbewältigung leisten ${ }^{15}$. Neben der Konkretisierung des Beitrags der Migrantinnen und Migranten an die Entwicklung ihrer Herkunftsländer sorgt das Ministerium vor allem auch dafür, dass die APD so eingesetzt wird, dass die Migrationsströme besser gesteuert werden können. Der grosszügig dotierte Haushalt des Ministeriums ist ein Beweis dafür, dass man sich nicht mit Lippenbekenntnissen begnügt, sondern Nägel mit Köpfen machen will. Verschiedene Ämter und Agenturen wurden dem neuen Ministerium unterstellt, so etwa die Agence nationale de l'accueil des étrangers et des migrations (Agentur für den Aufnahme von Ausländern und für Migration), die Grenzpolizei, die Sous-direction de la circulation des étrangers (Fremdenpolizei) usw. Zudem kann das Einwanderungsministerium bei der Erfüllung seines Auftrags auf die Unterstützung von Fachstellen zählen, die formell dem Aussenministerium angegliedert sind, darunter die Direction générale de la coopération internationale au développement DGCID (Direktion für internationale Entwicklungszusammenarbeit). Die DGCID ist verpflichtet, an allen vom neu geschaffenen Ministerium lancierten Entwicklungsprojekten mitzuwirken, welche die Migrantinnen und Migranten betreffen und/oder das Ziel verfolgen, einen Beitrag zur Migrationsbewältigung zu leisten. Das Budget 2008 des Ministeriums beläuft sich auf 639 Millionen Euro in Form von Zahlungskrediten und verteilt sich wie folgt auf die wichtigsten Politikbereiche: 49,9 Prozent für das Asylwesen, 29,6 Prozent für die Aufnahme und Integration von Ausländerinnen und Ausländern im Besitz einer Aufenthaltsgenehmigung, 13 Prozent für die Einwanderungspolitik, 7,2 Prozent für Stabsaufgaben, 4,5 Prozent für das codéveloppement und 0,2 Prozent für Einbürgerungen ${ }^{16}$. Obgleich das institutionelle Organigramm und die Mittelausstattung teilweise auf eine Neuzuweisung von Ressourcen zurückzuführen sind, die zuvor anderen Ministerien zugeordnet waren, so zeugt ihre Zusammenführung doch von einer pragmatischen Logik, die politische Richtungsentscheide begünstigt. Eine aufmerksame Betrachtung der einzelnen Elemente dieses Gefüges macht indessen deutlich, dass sich diese Logik stark an dem Ziel der Migrationssteuerung orientiert ${ }^{17}$.

\section{Massnahmen des codéveloppement: eine Typologie}

Beim Vollzug der französischen Politik des codéveloppement, die durch bilaterale Hilfeleistungen und die Beteiligung der Migrantinnen und Migranten

14 Décret no 2007-999 du 31 mai 2007, op. cit.

15 Ibid.

16 Das Programm „codéveloppement“ ist mit einem Zahlungskredit von 29 Millionen Euro und einem Verpflichtungskredit über 60 Millionen Euro dotiert. Budget 2008 du Ministère de l'immigration, de l'intégration, de l'identité nationale et du codéveloppement, <http://www.anaem.fr>.

17 C. Daum, op. cit. 
charakterisiert ist, kommen verschiedene Instrumente zum Einsatz, die sich vier grossen Handlungsfeldern zuordnen lassen: 1) Massnahmen zur Mobilisierung der Kompetenzen der Eliten der Diaspora; 2) lokale Infrastrukturprojekte, auf die die Migrantenvereinigungen grossen Wert legen; 3) Wiedereingliederungshilfe (dieser Begriff wird dem früher verwendeten Terminus „Rückkehrhilfe“ vorgezogen, weil er weniger abwertend konnotiert ist); 4) produktive Investitionen in den Herkunftsländern.

Um sicherzustellen, dass sich diese Instrumente sinnvoll ergänzen und eine koordinierte Migrationssteuerung erlauben, schliesst Frankreich bilaterale Rahmenabkommen über eine einvernehmliche Steuerung der Migrationsströme ab. Diese Abkommen verknüpfen die Entwicklungsfrage mit der Süd-NordMigration und sollen als Fundament für eine neue Partnerschaft zwischen Frankreich und den Entwicklungsländern dienen ${ }^{18}$. Das codéveloppement situiert sich an der Schnittstelle dreier zentraler Problemstellungen: internationale Migration, Globalisierung und Entwicklung. Die Herausforderungen, die diese drei Themenbereiche mit sich bringen, betreffen Industrie- und Entwicklungsländer gleichermassen. Tragfähige Lösungen können nur in einem dreiteiligen Dialog zwischen den beteiligten Ländern und den Migrantinnen und Migranten entwickelt werden. Voraussetzung dafür ist jedoch, dass sich die Migrationsbewältigung nicht mehr ausschliesslich auf den Vollzug einer nationalen Gesetzgebung und Reglementierung beschränkt, welche die Bedingungen für die Einreise und den Aufenthalt ausländischer Staatsbürger festlegen, sondern dass sie sich internationaler Verhandlungen bedient und sich in eine neue Sichtweise der APD einfügt. In diesem Sinne legen die Rahmenabkommen die Regeln der Nord-Süd-Zusammenarbeit mit den Entwicklungsländern partnerschaftlich fest mit dem Ziel, gemeinsam mit ihnen die legale Migration zu steuern, die illegale Einwanderung zu bekämpfen und Massnahmen des codéveloppement durchzuführen, welche dafür sorgen sollen, dass die Migration sowohl den Herkunftsals auch den Gastländern einen Nutzen bringt. Die Einbindung der Migrantinnen und Migranten und ihrer Vereinigungen an den Entwicklungsmassnahmen kann ferner ihre Integration in die französische Gesellschaft fördern und die Aufrechterhaltung enger Beziehungen zur Heimat begünstigen.

Diese Abkommen sind als ausbaufähige Verhandlungsgrundlage gedacht. Die Herkunftsländer legen Frankreich ihre Bedürfnisse dar und verpflichten sich im Gegenzug, gegen die illegale Migration vorzugehen. Zusätzlich zum erklärten Ziel, die Entwicklung der Herkunftsländer zu fördern, sollen diese Abkommen auch massgeblich zum Kampf gegen die illegale Einwanderung beitragen. Brice Hortefeux unterstreicht diese doppelte Zielsetzung wie folgt: „Die Herkunftsländer teilen Frankreich ihre Bedürfnisse mit und verpflichten sich, Frankreich bei der Bekämpfung der illegalen Einwanderung zu unterstützen. Als Gegenleistung nimmt Frankreich eine gewisse Zahl ihrer Staatsbürger auf und sorgt für die Ausbildung ihrer Eliten." ${ }^{19} \mathrm{Zu}$ diesem Zweck enthalten die Rahmenabkommen Bestimmungen zur Rückübernahme illegaler Einwanderer durch das Herkunftsland und sowie in der Regel auch über das codéveloppement, dessen

18 R. Munnich, Les accords de codéveloppement, un nouvel instrument de coopération avec le Sud, Paris, Haut Conseil de la coopération internationale, November 2007, <http://www.hcci.gouv.fr/ lecture/fiches/accord-de-co-developpement.html>.

19 B. Hortefeux, „Le codéveloppement, un enjeu majeur pour la France“, op. cit., eigene Übersetzung. 
Umfang von einem einen Indikator abhängig gemacht wird, der auf die Anzahl der Rückübernahmen abstellt. Die Absicht ist eindeutig: Die Bereitschaft der Herkunftsländer von Migrantinnen und Migranten, sich an der Migrationsbewältigung zu beteiligen, soll durch Entwicklungshilfeleistungen abgegolten werden ${ }^{20}$.

Die Instrumentalisierung der APD durch die französische Regierung zum Zwecke der Migrationsbewältigung verfolgt somit zwei Stossrichtungen, nämlich die Entwicklung auf der einen und die Kopplung der Hilfe an eine Gegenleistung auf der anderen Seite. Die erste Stossrichtung zielt auf die Finanzierung von Entwicklungsprogrammen in Regionen mit hohem Auswanderungsdruck $\mathrm{ab}$, um dort die Lebensbedingungen zu verbessern und Anreize für die Bevölkerung zu schaffen, in ihrer Heimat zu bleiben (beziehungsweise dorthin zurückzukehren), anstatt auszuwandern. Die zweite nennt als Voraussetzungen für die Gewährung von APD an die Länder des Südens den Kampf gegen die illegale Migration und die Wirksamkeit der diesbezüglichen Politiken.

\section{Codéveloppement: Fokussierung auf gemeinsame Interessen an der Migration}

Aber inwieweit ist die Instrumentalisierung der APD durch die französische Regierung sinnvoll? Welchen Nutzen und welche Risiken birgt diese Politik?

So wie es von der französischen Regierung präsentiert wird, dient das codéveloppement in erster Linie als Bezugsrahmen, der die gemeinsamen Interessen an der Migration in den Mittelpunkt stellt. Durch die Einbindung der Auswanderer in die Nord-Süd-Zusammenarbeit - über Investitionen und über die Bereitstellung ihres Fachwissens und Know-hows - erhalten die Entwicklungsländer Zugang zu Finanz- und Humanressourcen von unschätzbarem Wert. Bereits in der Vergangenheit haben Investitionen der Migrantenvereinigungen in den Herkunftsgemeinschaften viel bewirkt. Auch die im Rahmen des codéveloppement gewährte APD hat die Durchführung umfangreicher Entwicklungsprogramme mit enormem Nutzen für die lokalen Bevölkerungen ermöglicht. Aus der Sicht der Industrieländer fügt sich das codéveloppement nahtlos in die von ihnen gewählte Einwanderungspolitik ein. Die Instrumentalisierung der APD soll dazu dienen, die illegale Einwanderung zu bekämpfen und den Einwanderungsdruck abzuschwächen. So gesehen ist das codéveloppement nicht nur Ausdruck des Solidaritätsgedankens, der das Herzstück des republikanischen Erbes Frankreichs bildet, sondern auch ein wichtiges politisches Anliegen der Regierung, die den Bedenken der Bevölkerung im Zusammenhang mit Migrationsfragen Rechnung tragen will. Darin widerspiegelt sich die entwicklungspolitische Strategie Frankreichs, die die eigenen Interessen des Landes und jene seiner Partnerländer gleichermassen abzudecken versucht.

Mithilfe des codéveloppement, das einen Bogen zwischen der APD und dem Kampf gegen die illegale Einwanderung spannt, will Frankreich die Armut in den Entwicklungsländern verringern und gleichzeitig wirkungsvoller gegen die illegale Migration vorgehen. Nach Auffassung Frankreichs bietet dieser Politikbereich die Möglichkeit, die Zusammenarbeit zwischen Norden und Süden, aber auch zwischen Staat und Zivilgesellschaft zu intensivieren. Zumindest in der

20 C. Daum, op. cit. 
Theorie bildet das codéveloppement einen geeigneten Rahmen, um eine koordiniertes Vorgehen und eine Zusammenarbeit der Staaten in allen migrationsrelevanten Fragen zu gewährleisten. Die Verknüpfung von Entwicklungszusammenarbeit und Migrationsbewältigung kann ausserdem in den Industrienationen das Bewusstsein fördern, dass die Kontinente gegenseitig voneinander abhängig sind dass die Konsequenzen ungenügender Bemühungen zur Angleichung der Lebensstandards weltweit unweigerlich auf sie zurückfallen werden. Wird Ziel der Entwicklung der Herkunftsländer im Kampf gegen die Migration als ernsthaftes Ziel verfolgt, so könnte dies die Industrieländer des Nordens dazu bewegen, ihre bisherigen Politiken zu überdenken und sich vermehrt den Ländern des Südens zuzuwenden, um deren Entwicklungsbedürfnisse zu identifizieren und sie verstärkt zu berücksichtigen. Und geht man davon aus, dass die Armutsverringerung eine Eindämmung der Migrationsströme bewirkt, wie es im politischen Diskurs immer wieder behauptet wird, so müsste eine kohärente politische Strategie zur verbesserten Migrationsbewältigung logischerweise zu einer Aufstockung der APD und zu einer Neuausrichtung der Entwicklungshilfepolitik der Industrieländer führen.

Mit seinen Rahmenabkommen zur koordinierten Bewältigung der Migrationsströme und seiner Politik des codéveloppement verfügt Frankreich über ein wertvolles Instrument, um die Bereitschaft der afrikanischen Länder und der Zivilgesellschaft, gegen die illegale Migration vorzugehen, zu stärken ${ }^{21}$. Wird einem illegalen Einwanderer bei der Rückführung ein Mikrokredit für den Aufbau einer Existenzgrundlage gewährt, so wird dies von den Migrantinnen und Migranten selbst sowie von den Regierungen der Entwicklungsländer zweifellos positiver aufgenommen (und ist erst noch wirkungsvoller) als eine einfache Ausweisung. Ebenso erhöht die Durchführung von Entwicklungsprogrammen in den Entwicklungsländern deren Bereitschaft, ihre Migrationsströme besser zu steuern. Der Versuch, den Migrationsdruck durch die Realisierung eines gemeinsamen Ziels nämlich der Entwicklung - zu mildern, könnte somit die Zusammenarbeit zwischen Norden und Süden stärken. Dies kann aber nur gelingen, wenn diese Zusammenarbeit tatsächlich einem gemeinsamen Interesse dient.

\section{Eindämmung der Migration durch Entwicklung: kurzfristig illusorisch und wirkungslos}

Bevor die französische Politik des codéveloppement kritisiert wird, muss untersucht werden, inwiefern es ihr gelingt, die Menschen in den Entwicklungsländern von einer Auswanderung abzuhalten.

Die politische Strategie, den Migrationsdruck über die Entwicklungshilfe für die Länder des Südens zu mildern, scheint nicht nur ineffizient, sondern auch widersprüchlich. Die Idee, wonach das codéveloppement einen Beitrag zur Eindämmung der Migrationsströme leisten kann, mag an sich richtig sein - aber nur auf lange Sicht und unter der Voraussetzung, dass gewisse Bedingungen erfüllt sind.

Verschiedene Untersuchungen haben gezeigt, dass die Entwicklungsfortschritte der Länder des Südens den Auswanderungsdruck wohl verringern, aber nur sehr

21 C. Tasca, J. Pelletier et B. Barraux, Le codéveloppement à l'essai, Rapport d'information, $\mathrm{n}^{\circ} 417$ (2006-2007), Paris, Sénat, 25. Juli 2007. 
langsam. Bis diese Verringerung spürbar wird, können Jahrhunderte vergehen; ausserdem müssen sich dazu die Ungleichgewichte in Bezug auf die Verteilung des Reichtums auf nationaler wie auf internationaler Ebene massiv verringern ${ }^{22}$. Laut diesen Untersuchungen, die zu Beginn der 1970er-Jahre von Wilbur Zelinski (mobility transition model) lanciert und später von anderen Forschenden wie Ralph Rotte, Michael Vogler und Hein de Haas weitergeführt wurden, bewirkt der Entwicklungsprozess einer Gesellschaft in mehreren Etappen eine Veränderung der Auswanderungsneigung in der Bevölkerung. Die Zusammenhänge zwischen Entwicklung und internationaler Migration ändern sich mit der Zeit und lassen sich durch eine umgekehrte U-Kurve schematisch darstellen: Im Laufe der Entwicklung nimmt die Migration zunächst zu, um nach einer Phase der Stabilisierung wieder abzunehmen. Der Grund dafür lautet, dass sich im Zuge der Entwicklung die Ansprüche erhöhen und die Auswanderungsmöglichkeiten der Haushalte zunehmen, weil die finanziellen Hürden einer Auswanderung gesenkt werden, Arbeitsplätze in der Landwirtschaft verschwinden, wirtschaftliche Ungleichheiten zunehmen und das Human- und Sozialkapital gestärkt wird. Solche Veränderungen fördern die interne Migration in die Städte, wodurch sich die Migrationsströme verstärken und die individuellen Möglichkeiten zur Auswanderung zunehmen. Erst später, wenn die kombinierten Auswirkungen der demografischen Transition, der Produktivitätssteigerungen in der Landwirtschaft, der wirtschaftlichen Entwicklung und der Verringerung der Ungleichheiten spürbar werden, kommt es zu einem Versiegen und zuweilen gar zu einer Umkehr der Migrationsströme.

Die Frage, ob die Entwicklung zur Eindämmung der Migrationsströme beitragen kann, ist letztlich eine Frage des Zeithorizonts. Die Antwort lautet: Langfristig ja, allerdings nur unter gewissen Voraussetzungen; kurz- und mittelfristig nein. Jede Entwicklung schafft Ungleichgewichte. In ländlichen Regionen werden im Zuge der Entwicklung gesellschaftliche Beziehungen derart grundlegend verändert, dass es zu einer Landflucht kommt, die den Anfang aller internationalen Migrationsströme bildet. Eine Politik, die (kurzfristig) die Migration durch Entwicklung bekämpfen will, geht von der irrigen Annahme aus, dass potenzielle Migrantinnen und Migranten die Option einer Auswanderung effektiv ausklammern, nur weil sich der Lebensstandard in ihrem Land verbessert. Diese Hypothese geht nämlich von der absoluten Armut aus und lässt die Ungleichheiten und die tief greifenden Veränderungen, die durch die Entwicklung hervorgerufen werden, ausser Acht. Tatsächlich ist die Wahrscheinlichkeit gross, dass das codéveloppement während mehrerer Jahrzehnte eine Zunahme der Migrationsströme von Süden nach Norden bewirken wird. Wie lange diese Zunahme andauert, hängt vom ursprünglichen Entwicklungsstand des Landes sowie von seiner wirtschaftlichen und sozialen Entwicklung ab. Für Frankreich, dessen Einwanderer grösstenteils aus Afrika stammen, gilt diese Feststellung in besonderem Masse Angesichts der in Afrika herrschenden Armut wird deutlich, dass die Entwicklung des Kontinents für Frankreich vor allem im Bereich der illegalen Einwanderung problematisch werden dürfte. Zyniker würden daraus den

22 Siehe insbesondere R. Rotte and M. Vogler, The Effects of Development on Migration: Theoretical Issues and New Empirical Evidence, IZA Discussion Papers, Nr. 46, Bonn, Institute for the Study of Labor (IZA); sowie R.H. Adams, Jr. and J. Page, International Migration, Remittances, and Poverty in Developing Countries, World Bank Policy Research Working Paper, Nr. 3179, Washington, DC, World Bank. 
Schluss ziehen, es wäre zweckmässiger, Subsahara-Afrika in äusserster Armut $\mathrm{zu}$ belassen, anstatt seine Entwicklung zu fördern. Tatsache ist, dass sich der Migrationsdruck aus Afrika nur durch eine nachhaltige, grosszügige und langfristig ausgelegte Entwicklungspolitik verringern lässt. Bei der Verwirklichung des langfristigen Ziels, den Menschen in den Entwicklungsländern eine Existenzgrundlage zu bieten, die ihren Verbleib in der Heimat ermöglicht, muss eine Phase des erhöhten Migrationsdrucks in Kauf genommen werden. Zu glauben oder glauben zu machen -, man könne über das codéveloppement ganze Bevölkerungen im Verlaufe einiger politischer Amtsperioden an ihre Scholle binden, ist schlicht illusorisch.

\section{Grenzen des codéveloppement}

Abgesehen von dieser politischen Widersprüchlichkeit müssen mehrere Risiken und Grenzen bedacht werden, um die dem Konzept des codéveloppement und seiner Umsetzung in Frankreich zugrunde liegenden Problematiken auszuleuchten.

Seit jeher steht das codéveloppement in einem starken Spannungsfeld zwischen zwei Anliegen der öffentlichen Politik: Einerseits sollen die Migrantinnen und Migranten in ihren Bemühungen zur Entwicklung ihrer Herkunftsländer unterstützt werden, und andererseits sollen die Grenzkontrollen und die Massnahmen gegen die illegale Einwanderung gestärkt werden. Im ersten Fall geht es um die Bewältigung der Migrationsströme nach Europa durch die Förderung der Entwicklung der Herkunftsländer. Das erklärte Ziel - die Entwicklung - ist ein gemeinsames Anliegen der Länder des Südens wie des Nordens, auch wenn es die Letztgenannten lediglich als Mittel zur Erreichung eines übergeordneten politischen Zwecks betrachten, der darin besteht, den Migrationsdruck zu bewältigen. Im zweiten Fall geht es darum, die repressive Dimension der Migrationspolitik zu stärken, indem die Gewährung von Hilfe an Bedingungen geknüpft wird. Öffentliche Entwicklungshilfe wird nur gewährt, wenn gewisse politische Massnahmen ergriffen werden, die den Interessen Frankreichs entgegenkommen, und wenn gewisse Leistungsziele (z. B. die Rückübernahme illegaler Einwanderer) erfüllt werden.

Als Instrument der Entwicklungshilfe auf der einen und als Begleitmassnahme der französischen Sicherheitspolitik auf der anderen Seite birgt das codéveloppement einen inneren Widerspruch. Die Einbindung der Migrantinnen und Migranten in die Nord-Süd-Zusammenarbeit und deren Instrumentalisierung zum Zwecke einer wirtschaftlichen Entwicklung, die das Migrationsproblem lösen soll, unterstreichen die Zwiespältigkeit dieses stark politisch konnotierten Konzepts. Ausserdem ist eine Diskrepanz zwischen dem politischen Diskurs über das codéveloppement und seiner praktischen Umsetzung erkennbar: Obwohl eine eingehende Betrachtung der Aufgaben und des Budgets die Ausrichtung des codéveloppement auf die Migrationsbewältigung offensichtlich macht ${ }^{23}$, werden die Verfechter dieser Politik nicht müde zu betonen, dass das codéveloppement in keinerlei Zusammenhang mit sicherheitsrelevanten Einwanderungsfragen stehe und sein primäres Ziel nicht die Eindämmung der Einwanderung, sondern die

23 C. Daum, op. cit. 
Valorisierung der Beiträge der Migrantinnen und Migranten an die Entwicklung ihrer Herkunftsgemeinschaften sei. Dazu verweisen sie auf die drei Stärken (oder Vorzüge) des codéveloppement: Unterstützung der Initiativen von Migrantinnen und Migranten in ihrem Herkunftsland, Förderung der Investitionen der Migrantinnen und Migranten und Bekämpfung des brain drain. So unterstreicht ein kürzlich erschienener parlamentarischer Bericht zuhanden des Aussenministeriums, das codéveloppement dürfe keinesfalls als „Tarnung“ für die Rückschaffung von Migrantinnen und Migranten in ihr Herkunftsfall betrachtet werden, und es sei nicht seine Aufgabe, die Migrationsströme einzudämmen ${ }^{24}$. Die Realität sieht jedoch ganz anders aus: Die Entwicklungshilfe wird an Bedingungen geknüpft und gezielt auf Gebiete mit einem hohen Migrationsdruck ausgerichtet.

Aus politischer Sicht birgt diese Zwiespältigkeit für die Nord-Süd-Zusammenarbeit das Risiko, dass die Entwicklungsländer das codéveloppement ablehnen, weil sie befürchten, ihre Interessen würden nicht gebührend berücksichtigt. Die frühere Kulturministerin Malis, Aminata Dramane Traoré, vertritt die Ansicht, das codéveloppement sei unter den gegebenen Umständen im Grunde nichts anderes als ein Instrument der selektiven Einwanderungspolitik mit einem humanitären Anstrich, der dazu dienen soll, die Auswanderung zu bremsen und Einwanderern die Rückkehr schmackhaft zu machen ${ }^{25}$. In anderen Worten : Mit dem codéveloppement werde die eigentliche Absicht, die illegale Einwanderung zu bekämpfen, als Engagement zugunsten der Entwicklungsländer getarnt. Damit das codéveloppement zum Erfolg führt, müsste es nicht nur transparenter gestaltet, sondern auch als Mittel zur Wahrung gemeinsamer Interessen eingesetzt werden.

Ausserdem reicht das codéveloppement allein nicht aus, um effizient gegen den Migrationsdruck vorzugehen. Die Unzulänglichkeiten des Instrumentariums und des Mitteleinsatzes stehen in keinerlei Verhältnis zum erklärten Ziel, die Migration über Massnahmen des codéveloppement einzudämmen. Die Entwicklung kann nur dann eine Verringerung der Migrationsströme bewirken, wenn sie nachhaltig und langfristig konzipiert ist. Zwar weist Brice Hortefeux darauf hin, dass die Mittel für das codéveloppement unter der Präsidentschaft von Nicolas Sarkozy von drei auf 60 Millionen Euro aufgestockt wurden, was einer Erhöhung des französischen Budgets in diesem Bereich um das 20-fache entspricht ${ }^{26}$. Dieser Betrag reicht aber noch lange nicht aus. Zum Vergleich: Für die Grenzpolizei und die Rückführungen in das Herkunftsland stehen 20 Millionen Euro mehr zur Verfügung. Ausserdem müsste die Regierung ihre Hilfe drastisch aufstocken, um die illegale Einwanderung effektiv einzudämmen. 2007 betrug das Volumen der APD Frankreichs gerade 0,39 Prozent des Bruttoinlandprodukts das an der Konferenz von Monterrey festgelegte Ziel von 0,7 Prozent wurde also bei Weitem verfehlt. Und um die Migration an ihrem Ursprung einzudämmen, reicht selbst ein substanzielles codéveloppement nicht aus. Dazu müsste nämlich nicht nur die gesamte APD in ihren Grundzügen neu definiert werden (und zwar sowohl in Bezug auf den Mitteleinsatz als auch im Hinblick auf ihre

24 J. Godfrain et R. Cazenave, op. cit.

25 A.D. Traoré, Migrations africaines et violences: Où est le danger? Où est la vérité?, Bamako, Forum pour un autre Mali (FORAM), 17. Juni 2007, <http://www.co-developpement.org/?p=331>.

26 M. Joannidis, Le codéveloppement pourra-t-il freiner l'immigration?, Radio France Internationale, 1. Juli 2008, <http://www.rfi.fr/actufr/articles/102/article_67881.asp>. 
Durchführung), sondern auch die Handelspolitiken, denn nur so liessen sich die zahlreichen Konflikte ausräumen, die den Auswanderungsdruck in den Entwicklungsländern verursachen. Deshalb ist es illusorisch zu glauben, das codéveloppement sei für sich allein genommen eine Lösung zur Bewältigung des Migrationsphänomens ${ }^{27}$. Als Hauptargument für das codéveloppement eignet sich diese Behauptung jedenfalls nicht.

Die Instrumentalisierung der APD zum Zwecke der Migrationssteuerung schliesslich birgt die Gefahr, dass deren eigentliches Ziel - nämlich die Armutsverringerung - vernachlässigt wird. Gegenwärtig versucht Frankreich, das codéveloppement als äusserst pragmatischen Ansatz für seine APD zu etablieren, welcher über sektoral und geografisch abgegrenzte Massnahmen eine Eindämmung der Migrationsströme bewirken soll. Die APD soll schwerpunktmässig in den Herkunftsgebieten der Migrantinnen und Migranten ansetzen, indem Projekte durchgeführt werden, die Arbeitsplätze schaffen und so die Menschen an ihr Heimatland binden ${ }^{28}$. Ein solcher Ansatz steht jedoch im Widerspruch zur Grundprinzip der APD, nämlich dem Kampf gegen die Armut, und zwar ungeachtet der jeweiligen Region. Internationale Migrantinnen und Migranten kommen weder aus den ärmsten Ländern (der Grossteil kommt aus Entwicklungsländern mit mittlerem Einkommen), noch entstammen sie den ärmsten Bevölkerungsschichten (in der Regel verfügen sie über ein Einkommen oberhalb der Armutsschwelle $)^{29}$. Eine vermehrte Gewährung von Entwicklungsgeldern - die ohnehin knapp und zudem schlecht verteilt sind - unter dem Gesichtspunkt der Migration würde die bestehenden sozioökonomischen Ungleichheiten auf nationaler wie auf internationaler Ebene noch verstärken. Zudem stammen die Mittel für die Politik des codéveloppement unmittelbar aus dem Budget der APD, das von der DGCID verwaltet wird. Gewiss, die 60 Millionen Euro, die für das codéveloppement zur Verfügung stehen, bilden nur einen verschwindend kleinen Teil des gesamten APD-Haushalts Frankreichs. Falls dieser Anteil aber in Zukunft aufgestockt wird, muss unbedingt dafür gesorgt werden, dass die Mittel für das codéveloppement das Budget der APD nicht etwa ersetzen, sondern ergänzen. Eine Hilfs- und Entwicklungspolitik muss in erster Linie jenen Ländern zugute kommen, die es am meisten nötig haben. Nur so lassen sich die MillenniumsEntwicklungsziele erreichen. Wird nicht klar zwischen APD und codéveloppement unterschieden, könnte die französische Entwicklungshilfepolitik ihre angestammten Ziele in dem Masse aus den Augen verlieren, als die Mittel für das codéveloppement erhöht werden.

\section{Schlussfolgerungen}

Seit Beginn der 1980er-Jahre fand in der Politik eine schrittweise Annäherung zwischen den Problembereichen Entwicklung und Migration statt. Vor diesem Hintergrund ist die Entstehung des Konzepts des codéveloppement zu situieren.

$27 V$. Vanhaeverbeke, „Le codéveloppement, un concept qui se cherche encore“, Echos du COTA, dossier Quel partenariat entre ONG et migrants?, Nr. 110, Bruxelles, COTA asbl, März 2006, S. 3-5.

28 Allocution de la ministre déléguée à la Coopération, au Développement et à la Francophonie, $M^{m e}$ Brigitte Girardin, Conférence ministérielle euro-africaine sur la migration et le développement, Rabat, 10. Juli 2006

29 R.H. Adams, Jr. and J. Page, op. cit. 
In dem Masse, in dem sich die Einwanderungsfrage zu einem zentralen innenpolitischen Thema entwickelt hat, wuchs auch das Interesse am codéveloppement. Das Konzept entstand in Frankreich und vermochte sich dort mit der Errichtung des neuen Ministeriums für Einwanderungsfragen am raschesten zu institutionalisieren. Diese Entwicklung, die geprägt ist von einer Instrumentalisierung der APD zum Zwecke der Migrationssteuerung, ist Ausdruck des Willens der französischen Regierung, die Themenbereiche Migration und Entwicklungshilfe so miteinander zu verknüpfen, dass der Nutzen der Migration für die Entwicklung der Länder des Südens maximiert wird. Damit wird das Ziel verfolgt, die Menschen in den Entwicklungsländern von einer Auswanderung abzuhalten und durch eine an Bedingungen geknüpfte Gewährung von Entwicklungshilfe wirksamer gegen die illegale Einwanderung vorzugehen.

Auch auf der internationalen politischen Agenda gewinnt das codéveloppement an Bedeutung. Sehr rasch vermochte sich dieses Konzept auch ausserhalb Frankreichs durchzusetzen und fand Eingang in den Sprachgebrauch internationaler Organisationen. Dies gilt insbesondere für die Europäische Union, die sich seit dem Gipfel von Tampere im Jahr 1999 damit befasst. So äusserte das Europäische Parlament im Jahr 2004 sein Bedauern darüber, „dass die bisher vom Rat und den Mitgliedstaaten verabschiedeten Massnahmen zur Steuerung der Migrationsströme eher repressive als positive, zukunftsorientierte Massnahmen waren“, und erinnerte daran, dass „,Strategien zur Verringerung der Armut, zur Verbesserung der Lebens- und Arbeitsbedingungen, zur Schaffung von Arbeitsplätzen und zum Ausbau des Bildungswesens in den Herkunftsländern langfristig zur Normalisierung der Migrationsströme beitragen" ${ }^{\text {"30 }}$. Mit dem französischen EU-Vorsitz hat das codéveloppement einen zentralen Platz auf der politischen Agenda eingenommen. Brice Hortefeux unterstreicht dies mit den Worten, die Europäische Union habe die Beziehung zwischen Entwicklungshilfe und Kampf gegen die illegale Einwanderung zu lange ignoriert ${ }^{31}$. Die französische Regierung ist sich darüber im Klaren, dass die französischen Initiativen in diesem Bereich auf europäischer Ebene nicht isoliert bleiben dürfen, und will deshalb die Zusammenarbeit mit der Europäischen Kommission und den anderen EU-Mitgliedstaaten auf dem Gebiet der Steuerung der Migrationsströme und des codéveloppement vertiefen $^{32}$. Dieser Wille wird insbesondere im neuen Europäischen Migrationspakt konkretisiert, der spezifische Bestimmungen über das codéveloppement enthält.

Angesichts des Siegeszuges des codéveloppement erscheint es heute wichtiger denn je, die Zweckmässigkeit des Konzepts zu analysieren, seine Tragweite zu definieren und seine Vorzüge und Grenzen in Bezug auf seine Wirksamkeit zu beurteilen. Wie am Beispiel Frankreichs dargelegt wurde, bietet das codéveloppement die Möglichkeit, die Migrationsproblematik unter dem Gesichtspunkt gemeinsamer Interessen anzugehen und so die Nord-Süd-Zusammenarbeit zu stärken. In diesem Sinn ist das Konzept als wichtige politische Innovation zu bewerten. Allerdings zeigen sich bei seiner Umsetzung gewisse Grenzen und Ungereimtheiten, die nicht ausser Acht gelassen werden dürfen: Erstens ist der

30 Europäisches Parlament, Entschliessung des Europäischen Parlaments zu den Zusammenhängen zwischen legaler und illegaler Migration und Integration der Migranten (2004/2137), 2004.

31 B. Hortefeux, „Le codéveloppement, un enjeu majeur pour la France“, op. cit.

32 Conseil des ministres, Les accords de gestion concertée des flux migratoires et de codéveloppement, communiqué du Conseil des ministres, 29. August 2007. 
Versuch, den Migrationsdruck über die Entwicklungshilfe für die Länder des Südens abzuschwächen, zumindest kurzfristig eine ineffiziente und äusserst widersprüchliche politische Strategie. Zweitens bleibt das Konzept des codéveloppement trotz aller Definitionsversuche zwiespältig und vermag sich nicht aus dem Spannungsfeld zwischen Entwicklung der Herkunftsländer und Bekämpfung der illegalen Einwanderung zu lösen. Für Frankreich birgt dies die Gefahr, dass das codéveloppement von den Entwicklungsländern und von den Migrantenkreisen als Tarnung für eine einseitige Interessenpolitik wahrgenommen wird. Drittens reicht das codéveloppement für sich allein genommen nicht aus, um den Migrationsdruck zu verringern. Nur ein rasches und anhaltendes Wirtschaftswachstum in allen Entwicklungsländern - welches durch eine Aufstockung der Budgets für das codéveloppement und die APD, durch eine Hinterfragung der Handelspolitiken der Industrieländer und durch entschlossene Bemühungen zur Lösung gegenwärtiger Konflikte gewährleistet werden muss kann die illegale Migration nachhaltig verringern. Und letztlich birgt die Instrumentalisierung der APD für die Zwecke der Migrationssteuerung die Gefahr, dass das eigentliche Ziel der Entwicklungszusammenarbeit - die Armutsbekämpfung - vernachlässigt wird.

Das codéveloppement versteht sich als Rahmen für eine dreiteilige Zusammenarbeit, die den Nutzen der Migration für die Entwicklung der Länder des Südens stärken und die Bewältigung der Migrationsströme in die Industrieländer fördern soll. Aber um dieses Ziel zu erreichen, müssen die Industrienationen eine ehrgeizige Politik der Entwicklungszusammenarbeit und des codéveloppement ausarbeiten, das codéveloppement in den Dienst gemeinsamer Interessen stellen und eine deutliche Unterscheidung treffen zwischen der klassischen Entwicklungszusammenarbeit und Massnahmen zur Migrationsbewältigung. Ansonsten könnte das codéveloppement im Stadium eines ambitionierten politischen Projekts verharren oder gar das Gegenteil dessen bewirken, was eigentlich beabsichtigt war.

\section{Bibliographie}

Adams, R.H. Jr. and J. Page, International Migration, Remittances, and Poverty in Developing Countries, World Bank Policy Research Working Paper, Nr. 3179, Washington, DC, World Bank.

Cazenave, R. et J. Godfrain, Rapport sur le codéveloppement, rapport envoyé au Ministère des affaires étrangères de la France, Januar 2007, <http://www.richardcazenave.com/media/Rapport_codeveloppement. pdf $>$.

Conseil des ministres, Les accords de gestion concertée des flux migratoires et de codéveloppement, communiqué du Conseil des ministres, 29. August 2007.

Daum, C., „Le codéveloppement, grandeur et décadence d'une aspiration généreuse“, Revue internationale et stratégique, N. 4 (68), 2007, S. 49-59.

Décret no 2007-999 du 31 mai 2007 relatif aux attributions du ministre de l'Immigration, de l'Intégration, de l'Identité nationale et du Codéveloppement, Journal officiel de la République française, Nr. 125, 1. Juni, S. 9964, Text Nr. 11.

Europäisches Parlament, Entschliessung des Europäischen Parlaments zu den Zusammenhängen zwischen legaler und illegaler Migration und Integration der Migranten (2004/2137), 2004.

Guengant, J.-P., „Quel lien entre migrations internationales et développement ?“, Projet, dossier Migrations et frontières, Nr. 272, La Plaine Saint-Denis, Centre d'étude et de recherche en action sociale (CERAS), Dezember 2002.

Haas, H. de, Turning the Tide? Why „Development Instead of Migration“ Policies are Bound to Fail, Oxford, International Migration Institute, University of Oxford, 2006. 
Hortefeux, B., „Le codéveloppement, un enjeu majeur pour la France“, Les Echos, 31. August 2007.

Joannidis, M., Le codéveloppement pourra-t-il freiner l'immigration?, Radio France Internationale, 1. Juli 2008, <http://www.rfi.fr/actufr/articles/102/article_67881.asp>.

Ministère des affaires étrangères (MEA), Le codéveloppement: présentation générale, Paris, MEA, 26. Januar 2005.

Munnich, R., Les accords de codéveloppement, un nouvel instrument de coopération avec le Sud, Paris, Haut Conseil de la coopération internationale, November 2007, <http://www.hcci.gouv.fr/lecture/ fiches/accord-de-co-developpement.html>.

Rotte, R. and M. Vogler, The Effects of Development on Migration: Theoretical Issues and New Empirical Evidence, IZA Discussion Papers, Nr. 46, Bonn, Institute for the Study of Labor (IZA).

Stalker, P., „Migration Trends and Migration Policy in Europe“, International Migration, vol. 40, Nr. 5, S. $151-179$.

Tasca, C., J. Pelletier et B. Barraux, Le codéveloppement à l'essai, Rapport d'information, no 417 (20062007), Paris, Sénat, 25. Juli 2007.

Traoré, A.D., Migrations africaines et violences: Où est le danger? Où est la vérité?, Bamako, Forum pour un autre Mali (FORAM), 17. Juni, <http://www.co-developpement.org/?p=331>.

Vanhaeverbeke, V., „Le codéveloppement, un concept qui se cherche encore“, Echos du COTA, dossier Quel partenariat entre ONG et migrants?, Nr. 110, Bruxelles, COTA asbl, März 2006, S. 3-5. 